\title{
Bounds on real finite linear groups that preserve a symmetric or skew symmetric bilinear form
}

\author{
Michael J. Collins \\ Communicated by Robert M. Guralnick
}

\begin{abstract}
We extend the results of our earlier work on Jordan-type bounds for finite subgroups of complex classical groups to real groups. The bounds that we obtain are related to our previous results by means of structural results for finite linear groups that we can generalise here to compact groups. For the real analogue of orthogonal groups, we take into account the signature of a real quadratic form to determine bounds in every case.
\end{abstract}

\section{Introduction}

In [2] and [3], we obtained precise bounds (in Jordan's sense) for finite subgroups of the general linear group $\operatorname{GL}(n, \mathbb{C})$, results that were later extended to cover finite subgroups of the complex classical groups [5]. Here we consider equivalent questions for finite subgroups of $\operatorname{GL}(n, \mathbb{R})$, where we seek tighter bounds.

Let $V$ be a finite-dimensional real vector space equipped with a (possibly trivial) symmetric or skew symmetric bilinear form B, and let $G$ be a finite group that acts on $V$ and preserves the form B. Recall that the radical of the bilinear form B is the subspace

$$
\operatorname{Rad}(\mathrm{B})=\{u \in V \mid \mathrm{B}(u, v)=0 \text { for all } v \in V\},
$$

and this is clearly $G$-invariant. So, by Maschke's theorem, $\operatorname{Rad}(\mathrm{B})$ has a $G$-invariant complement $V_{0}$ in $V$, and the restriction $\mathrm{B}_{0}$ of $\mathrm{B}$ to $V_{0}$ is nondegenerate. If $\mathrm{B}$ is skew symmetric, then necessarily $\operatorname{dim}\left(V_{0}\right)$ is even. In the case that $\mathrm{B}$ (and hence also $\left.\mathrm{B}_{0}\right)$ is symmetric, there is an associated quadratic form $\mathrm{Q}$ given by $\mathrm{Q}(v)=\mathrm{B}(v, v)$, and it is here that the real case differs from working with complex linear groups since the form $\mathrm{Q}$ has a signature as well as a rank (which is just $\operatorname{dim}\left(V_{0}\right)$ ). To fix notation, suppose that the rank is $p+q$ and the signature $p-q$.

Throughout, we let $f(n)$ and $g(n)$ denote the "Jordan bounds" obtained in [2] or [5], namely the bounds on the minimal index of an abelian normal subgroup in an arbitrary finite subgroup of $\mathrm{GL}(n, \mathbb{C})$ or $\mathrm{O}(n, \mathbb{C})$, respectively. 
Theorem A. Let $V, G$ and $\mathrm{B}$ be as described above. Put $\operatorname{dim}(\operatorname{Rad}(\mathrm{B}))=k$ and, in the case that $\mathrm{B}$ is skew symmetric, put $\operatorname{dim}\left(V_{0}\right)=2 m$. Then $G$ has an abelian normal subgroup A such that

(i) if $\mathrm{B}$ is skew symmetric, then $[G: A] \leq g(k) \cdot f(m)$,

(ii) if $\mathrm{B}$ is symmetric, then $[G: A] \leq g(k) \cdot g(p) \cdot g(q)$.

In particular, if $G$ is any finite subgroup of $\mathrm{GL}(n, \mathbb{R})$, then $[G: A] \leq g(n)$.

Remarks. (1) Generically, the bounds $f(n)$ and $g(n)$ take the value $(n+1)$ ! when $n \geq 71$ and $n \geq 25$, respectively, and these are achieved by the (real) $n$-dimensional representations of the symmetric groups $S_{n+1}$. We refer the reader to [2,5] for the values of these bounds for smaller values of $n$ and for a description of the groups that achieve those bounds.

(2) It is well known that any complex representation of a finite group is equivalent to a unitary representation, and similarly that any real representation of a finite group is equivalent (over $\mathbb{R}$ ) to an orthogonal representation. Furthermore, it is essentially part of the derivation of the Frobenius-Schur indicator that any finite subgroup of the complex orthogonal group $\mathrm{O}(n, \mathbb{C})$ is conjugate in $\operatorname{GL}(n, \mathbb{C})$ to a subgroup of $\mathrm{O}(n, \mathbb{R}) .{ }^{1}$ Thus the final statement of Theorem $\mathrm{A}$ is a direct consequence of the results for the orthogonal case in [5]. Indeed, we use this observation to obtain the factor $g(k)$ in the theorem.

We will prove Theorem A by establishing a structural theorem for a $G$-invariant decomposition of $V$ from which such bounds can be determined immediately. This result does not depend on finiteness and extends to compact groups, so we will state and prove it in this slightly greater generality. The bounds, however, do depend on the application of the classification of finite simple groups to determine bounds for primitive linear groups in [3].

Our bound when B is skew symmetric uses the following result that may be of independent interest. Here, too, we may suppose that our group is merely compact rather than finite.

Theorem B. Let $G$ be a compact group. Then $G$ can be embedded in $\operatorname{Sp}(2 n, \mathbb{R})$ if and only if it can be embedded in $\operatorname{GL}(n, \mathbb{C})$.

The main structural decomposition theorem is the following.

1 Throughout, we will reserve the term orthogonal group and the notation $\mathrm{O}(n, \mathbb{R})$ for the group of real $n \times n$ matrices that preserve the quadratic form $x_{1}^{2}+\cdots+x_{n}^{2}$, namely the group $\left\{X \mid X^{T} X=I\right\}$. 
Theorem C. Let $V$ be a finite-dimensional real vector space equipped with a symmetric or skew symmetric bilinear form $\mathrm{B}$, and let $\mathrm{G}$ be a compact group that acts on $V$ and preserves the form $\mathrm{B}$.

(i) $V$ admits a $G$-invariant decomposition $V=\operatorname{Rad}(\mathrm{B}) \oplus V_{0}$, where $\mathrm{B}_{0}=\left.\mathrm{B}\right|_{V_{0}}$ is nondegenerate. This decomposition is unique if and only if $\operatorname{Rad}(\mathrm{B})$ and $V_{0}$ have no irreducible $G$-invariant constituent in common.

(ii) If $\mathrm{B}$ is symmetric, then $V_{0}$ has a $G$-invariant decomposition $V_{0}=V_{p} \oplus V_{q}$, where $V_{p}$ and $V_{q}$ are subspaces of dimensions $p$ and $q$ on which the restrictions of $\mathrm{B}$ are positive and negative definite respectively, and where $V_{p}$ and $V_{q}$ are mutually orthogonal with respect to $\mathrm{B}$.

We will prove Theorem B in Section 2 and then embark on the proof of Theorem C, first proving part (i) in Section 3 and then, by studying invariant quadratic forms in Section 4, obtain the finer decomposition of part (ii). These results are applied to prove Theorem A in Section 5.

\section{The proof of Theorem B}

We start with a classical result that establishes the result in one direction.

Proposition 1. Let $G$ be a compact group, and let $\mathrm{X}$ be a complex matrix representation of $G$. Then the matrix representation

$$
\hat{\mathrm{X}}: g \mapsto\left(\begin{array}{cc}
\mathrm{X}(g) & 0 \\
0 & \mathrm{X}(g)
\end{array}\right)
$$

is similar to a representation by real symplectic matrices.

Proof. First we may assume ${ }^{2}$ without loss that the matrix representation $\mathrm{X}$ is unitary. Then the representation $\hat{\mathrm{X}}$ is realisable over $\mathbb{R}$ since

$$
\begin{aligned}
\left(\begin{array}{cc}
\frac{1}{\sqrt{2}} I & \frac{i}{\sqrt{2}} I \\
\frac{i}{\sqrt{2}} I & \frac{1}{\sqrt{2}} I
\end{array}\right) \cdot\left(\begin{array}{cc}
\mathrm{X} & 0 \\
0 & \overline{\mathrm{X}}
\end{array}\right) \cdot\left(\begin{array}{cc}
\frac{1}{\sqrt{2}} I & \frac{-i}{\sqrt{2}} I \\
\frac{-i}{\sqrt{2}} I & \frac{1}{\sqrt{2}} I
\end{array}\right) & =\left(\begin{array}{cc}
\frac{1}{2}(\mathrm{X}+\overline{\mathrm{X}}) & \frac{-i}{2}(\mathrm{X}-\overline{\mathrm{X}}) \\
\frac{i}{2}(\mathrm{X}-\overline{\mathrm{X}}) & \frac{1}{2}(\mathrm{X}+\overline{\mathrm{X}})
\end{array}\right) \\
& =\left(\begin{array}{cc}
\mathrm{A} & \mathrm{B} \\
-\mathrm{B} & \mathrm{A}
\end{array}\right),
\end{aligned}
$$

where we suppress $g$ and write $\mathrm{X}=\mathrm{X}(g)=\mathrm{A}+i \mathrm{~B}$ for real matrices $\mathrm{A}$ and $\mathrm{B}$. Furthermore, since $\mathrm{X}$ is a unitary matrix, $\mathrm{A}^{T} \mathrm{~A}+\mathrm{B}^{T} \mathrm{~B}=I$ and $\mathrm{A}^{T} \mathrm{~B}=\mathrm{B}^{T} \mathrm{~A}$. (Here, as below, $I$ is the $n \times n$ identity matrix.)

\footnotetext{
${ }^{2}$ We need only replace the usual averaging process for finite groups by an integral with respect to Haar measure. See comments made in [9, Chapter 4].
} 
Now consider the representation

$$
\tilde{\mathrm{X}}: g \mapsto\left(\begin{array}{rr}
\mathrm{A}(g) & \mathrm{B}(g) \\
-\mathrm{B}(g) & \mathrm{A}(g)
\end{array}\right) .
$$

Again, suppressing $g$ for clarity, we have

$$
\left(\begin{array}{ll}
\mathrm{A}^{T} & -\mathrm{B}^{T} \\
\mathrm{~B}^{T} & \mathrm{~A}^{T}
\end{array}\right) \cdot\left(\begin{array}{rr}
0 & I \\
-I & 0
\end{array}\right) \cdot\left(\begin{array}{rr}
\mathrm{A} & \mathrm{B} \\
-\mathrm{B} & \mathrm{A}
\end{array}\right)=\left(\begin{array}{rr}
0 & I \\
-I & 0
\end{array}\right)
$$

so that the real matrix representation $\tilde{X}$ preserves a nondegenerate real skew symmetric form.

Remark. The proof actually shows that $\mathrm{U}(n, \mathbb{C})$ can be embedded into $\operatorname{Sp}(2 n, \mathbb{R})$. This was probably known to Frobenius and Schur. We include this result explicitly because we use it in establishing the converse.

Proposition 2. Let $G$ be an irreducible compact subgroup of $\operatorname{Sp}(2 n, \mathbb{R})$, and let $M$ be the corresponding $\mathbb{R} G$-module. Then $\mathbb{C} \otimes M=L \oplus L^{*}$ is the direct sum of two simple $\mathbb{C} G$-modules that are the dual to each other (but possibly self-dual).

Proof. $\mathbb{C} \otimes M$ cannot be simple, for then it would afford an irreducible representation of $G$ that is realisable over $\mathbb{R}$ and hence ${ }^{3}$ of Frobenius-Schur indicator +1 , contrary to the fact that $G$ is a subgroup of $\operatorname{Sp}(2 n, \mathbb{C})$. Now let $L$ be a simple submodule of $\mathbb{C} \otimes M$ of minimal dimension, and let $L^{*}$ be its dual (possibly isomorphic to $L$ ). Then $L^{*} \cong \bar{L}$ and, by Proposition $1, L \oplus L^{*} \cong \mathbb{C} \otimes \hat{L}$ for some $\mathbb{R} G$-module $\hat{L}$ of dimension $2 m$, where $m=\operatorname{dim} L \leq n$.

Now

$$
\begin{aligned}
\operatorname{Hom}_{\mathbb{C} G}(L, \mathbb{C} \otimes M) & \subseteq \operatorname{Hom}_{\mathbb{C} G}\left(L \oplus L^{*}, \mathbb{C} \otimes M\right) \\
& =\operatorname{Hom}_{\mathbb{C} G}(\mathbb{C} \otimes \hat{L}, \mathbb{C} \otimes M)
\end{aligned}
$$

while, by [6, equation (29.5)], letting $A$ be the $\mathbb{R}$-subalgebra of the matrix algebra $\mathcal{M}_{n}(\mathbb{R})$ spanned by the elements of $G$, we have

$$
\operatorname{Hom}_{\mathbb{C} \otimes A}(\mathbb{C} \otimes \hat{L}, \mathbb{C} \otimes M) \cong \mathbb{C} \otimes \operatorname{Hom}_{A}(\hat{L}, M) .
$$

\footnotetext{
${ }^{3}$ Here we use the language of finite groups, but the arguments on invariant forms extend verbatim provided that we refer to the kind of the representation. We have used the term indicator and its value as this is more familiar to finite group theorists. See [9, Theorem 31 and the subsequent Remark (1)].
} 
Since $L \subseteq \mathbb{C} \otimes M$, it follows that

$$
\operatorname{Hom}_{\mathbb{R} G}(\hat{L}, M) \neq 0 .
$$

But $M$ is simple, so $\hat{L} \cong M$, and hence $L \oplus L^{*} \cong \mathbb{C} \otimes \hat{L} \cong \mathbb{C} \otimes M$.

Corollary 3. Under the hypothesis of Proposition 2, there is an embedding of $G$ into $\mathrm{GL}(n, \mathbb{C})$.

Proof. $L$ and $L^{*}$ afford representations of $G$ having the same kernel, which is therefore trivial.

Proof of Theorem B. One direction is given by Proposition 1. So let $G$ be a compact subgroup of $\operatorname{Sp}(2 n, \mathbb{R})$, and let $M$ be the corresponding $\mathbb{R} G$-module, endowed with a real $G$-invariant skew symmetric form. We proceed by induction on $\operatorname{dim} M$, noting that we have an embedding of $G$ into $\operatorname{GL}(n, \mathbb{C})$ if $M$ is simple, by Corollary 3. So we suppose that $M$ is not simple.

Let $L$ be a simple submodule of $M$. Since the skew symmetric form on $M$ restricts to $L$, either $\operatorname{Rad} L=L$ or $\operatorname{Rad} L=0$, where $\operatorname{Rad} L$ denotes the radical of the restriction of the form to $L$.

In the first case, $L \subseteq L^{\perp}$, where orthogonality is defined with respect to the form on $M$. We have $M / L^{\perp} \cong L^{*}$, where $L^{*}$ is dual to $L$ (cf. [4, Lemma 1], which holds in the real case too). So we have, applying Maschke's theorem (which, as before, extends to compact groups - see footnote 2), $M=L^{\perp} \oplus L^{*}$, where the kernels of the actions of $L$ and $L^{*}$ are the same. Now write $L^{\perp}=L \oplus N$ as an $\mathbb{R} G$-module. Since $\operatorname{dim} L+\operatorname{dim} L^{\perp}=\operatorname{dim} M$ and $L \subseteq L^{\perp}$, we deduce that $\operatorname{Rad}\left(L^{\perp}\right)=L$ and that the restriction of the skew symmetric form to $N$ is nondegenerate. So we have an embedding

$$
G \hookrightarrow \mathrm{GL}(L) \times \mathrm{Sp}(N)
$$

and hence, by induction, embeddings

$$
G \hookrightarrow \operatorname{GL}(m, \mathbb{R}) \times \operatorname{GL}(n-m, \mathbb{C}) \hookrightarrow \operatorname{GL}(n, \mathbb{C}),
$$

where $\operatorname{dim} L=\operatorname{dim} L^{*}=m$ and $\operatorname{dim} N=2(n-m)$.

If $\operatorname{Rad} L=0$, then $M=L \oplus L^{\perp}$ and also $\operatorname{Rad} L^{\perp}=0$. So we also have an embedding

$$
G \hookrightarrow \operatorname{Sp}(L) \times \operatorname{Sp}\left(L^{\perp}\right)
$$

and, again by induction, a series of embeddings

$$
G \hookrightarrow \operatorname{GL}\left(m_{1}, \mathbb{C}\right) \times \operatorname{GL}\left(m_{2}, \mathbb{C}\right) \hookrightarrow \operatorname{GL}(n, \mathbb{C}),
$$

where $\operatorname{dim} L=2 m_{1}$ and $\operatorname{dim} L^{\perp}=2 m_{2}$.

This completes the proof of Theorem B. 
Remark. The results of this section give a simple and direct proof in our situation of the general fact, first shown by E. Cartan, that a connected Lie group has a unique conjugacy class of maximal compact subgroups. For a proof of this, see either [7, Theorem 3.1 of Chapter XV (and comments in the preface)] or [1] together with references therein. See also [8, Chapter X, in particular Section 2 and Table IV of Section 6] for a complete description of all the groups involved.

\section{The radical and its complement}

We assume the hypothesis of Theorem $\mathrm{C}$, namely that $V$ is a finite-dimensional real vector space equipped with a symmetric or skew symmetric bilinear form B and that $G$ is a compact group that acts on $V$ and preserves the form B. In this section, we prove part (i) of the theorem.

Maschke's theorem extends as before so that $\operatorname{Rad}(\mathrm{B})$ has a $G$-invariant complement $V_{0}$ and the restriction of $\mathrm{B}$ to $V_{0}$ is nondegenerate since $\operatorname{Rad}\left(\left.\mathrm{B}\right|_{V_{0}}\right) \subseteq \operatorname{Rad}(\mathrm{B})$. If $\operatorname{Rad}(\mathrm{B})$ and $V_{0}$ had a constituent in common, then they would have simple submodules $L$ and $L_{0}$ that were isomorphic. Let $\vartheta: L_{0} \rightarrow L$ be an isomorphism. Then, if $V_{0}=L_{0} \oplus N_{0}$, where $N_{0}$ is $G$-invariant, and we put

$$
\hat{L}_{0}=\left\{l+\vartheta(l) \mid l \in L_{0}\right\},
$$

a different $G$-invariant complement to $\operatorname{Rad}(\mathrm{B})$ is given by $\hat{V}_{0}=\hat{L}_{0} \oplus N_{0}$. Conversely, a different complement $V_{1}$ projects nontrivially into $V / V_{0}$ and so shares a constituent with $\operatorname{Rad}(B)$. This completes the proof of part (i).

Since any real representation of $G$ is equivalent to an orthogonal representation, if $\operatorname{dim}(\operatorname{Rad}(\mathrm{B}))=k$, the action of $G$ on $\operatorname{Rad}(\mathrm{B})$ can be embedded in $\mathrm{O}(k, \mathbb{R})$. To prove part (ii), and more generally to prove Theorem $A$ in the skew symmetric case, we can effectively confine our attention to $V_{0}$ or, equivalently, assume that the form $\mathrm{B}$ is nondegenerate.

\section{Invariant symmetric bilinear forms}

Throughout this section, B will denote a nondegenerate symmetric bilinear form on a real vector space $V$. We have the associated quadratic form

$$
\mathrm{Q}(v)=\mathrm{B}(v, v),
$$

from which B may be recovered via the formula

$$
\mathrm{B}(u, v)=\frac{1}{2}(Q(u+v)-Q(u)-Q(v)) .
$$

We now fix notation, and work under the following hypothesis. 
Hypothesis I. $V$ is a finite-dimensional real vector space endowed with a nondegenerate quadratic form $\mathrm{Q}$ and associated symmetric bilinear form $\mathrm{B}$, and $G$ is a compact group that acts on $V$ and preserves $\mathrm{Q}$ (or, equivalently, B).

Proposition 4. Suppose that $G$ acts irreducibly. Then $\mathrm{Q}$ is either positive or negative definite.

Proof. Fix a basis for $V$ corresponding to a representation $\sigma$ of $G$ by orthogonal matrices, and let $B$ be the real symmetric matrix that then represents B. Since B is $G$-invariant,

$$
\sigma(x)^{T} B \sigma(x)=B
$$

for all $x \in G$. For any nonzero eigenvalue $\lambda$ of $B$, we have

$$
(B-\lambda I) \sigma(x)=\sigma(x)(B-\lambda I)
$$

for all $x \in G$. Since $\sigma$ is irreducible, $B-\lambda I$ is either zero or invertible by Schur's lemma; hence $B=\lambda I$, and the quadratic form $\mathrm{Q}$ is either positive or negative definite, depending on the sign of $\lambda$.

Remark. The proof of Proposition 4 actually establishes uniqueness of the forms, up to scalar multiples.

Recall that a subspace $U$ of $V$ is said to be totally isotropic if $\mathrm{B}\left(u, u^{\prime}\right)=0$ for all $u, u^{\prime} \in U$ or, equivalently, if $\mathrm{Q}(u)=0$ for all $u \in U$. We will require the following two lemmas to complete the proof of Theorem $\mathrm{C}$.

Lemma 5. Not every irreducible $G$-invariant subspace of $V$ is totally isotropic.

Proof. Suppose to the contrary that every irreducible $G$-invariant subspace of $V$ is totally isotropic; pick one, say $U$, of minimal dimension. Then, working with the associated bilinear form $\mathrm{B}$, the subspace

$$
U^{\perp}=\{v \in V \mid \mathrm{B}(u, v)=0 \text { for all } u \in U\}
$$

is $G$-invariant and $U \subseteq U^{\perp} \neq V$ since $U$ is totally isotropic and Q is nondegenerate.

Let $\hat{U}$ be a $G$-invariant complement to $U^{\perp}$ in $V$. Then there is a natural injection $\varphi$ from $\hat{U}$ into the dual space of $U$ defined by $\varphi(\hat{u})(u)=\mathrm{B}(u, \hat{u})$ for all $u \in U$, $\hat{u} \in \hat{U}$; furthermore, since $U$ was chosen of minimal dimension, $G$ acts irreducibly on $\hat{U}$ so that $\hat{U}$ is also totally isotropic and $\varphi$ is an isomorphism.

Without loss, we may now suppose that $V=U \oplus \hat{U}$. Pick a basis for $U$ with respect to which $G$ acts by orthogonal matrices. Take the "dual basis" for $\hat{U}$. Since B 
is then represented by the matrix

$$
\left(\begin{array}{ll}
0 & I \\
I & 0
\end{array}\right)
$$

if the actions of $G$ on $U$ and $\hat{U}$ are given by matrices $A(g)$ and $\hat{A}(g)$, respectively, we have the matrix equation

$$
\left(\begin{array}{cc}
A(g) & 0 \\
0 & \hat{A}(g)
\end{array}\right)^{T} \cdot\left(\begin{array}{cc}
0 & I \\
I & 0
\end{array}\right) \cdot\left(\begin{array}{cc}
A(g) & 0 \\
0 & \hat{A}(g)
\end{array}\right)=\left(\begin{array}{cc}
0 & I \\
I & 0
\end{array}\right),
$$

whence $\hat{A}(g)=\left(A(g)^{T}\right)^{-1}=A(g)$ for all $g \in G$. So the actions of $G$ on $U$ and on $\hat{U}$ are equivalent.

Now let $\vartheta: U \rightarrow \hat{U}$ be the linear transformation defined by mapping the chosen basis of $U$ to its dual basis in $\hat{U}$. Then, for a basis element $u_{i} \in U$,

$$
\mathrm{B}\left(u_{i}+\vartheta\left(u_{i}\right), u_{i}+\vartheta\left(u_{i}\right)\right)=2
$$

so that the $G$-invariant subspace

$$
\{u+\vartheta(u) \mid u \in U\}
$$

is irreducible but not totally isotropic, a contradiction.

Lemma 6. There exists a direct sum decomposition of Vinto irreducible G-invariant subspaces that are mutually orthogonal with respect to the associated bilinear form $\mathrm{B}$, and on each of which the restriction of $\mathrm{Q}$ is nonzero.

Proof. By Lemma 5, there exists an irreducible $G$-invariant subspace $U$ on which the restriction of B is nonzero. Put $W=U^{\perp}$, where orthogonality is defined with respect to $\mathrm{B}$. Then $V=U \oplus W$ and $\operatorname{Rad}_{W}\left(\left.\mathrm{~B}\right|_{W}\right)=0$, so we may repeat this process recursively.

Proof of Theorem C (ii). Decompose the subspace $V_{0}$ using Lemma 6. By Proposition 2, the restriction of Q to each irreducible summand of $V_{0}$ is either positive or negative definite, and we collect $V_{p}$ and $V_{q}$ as the two relevant direct sums to obtain the desired decomposition of $V$. This establishes the orthogonality claim too.

\section{Bounds for finite groups}

We start with an easy lemma.

Lemma 7. Let $V$ be a finite-dimensional vector space $V$, and let $G$ be a finite subgroup of $\mathrm{GL}(V)$. Suppose that there is a $G$-invariant decomposition $V=V_{1} \oplus V_{2}$, 
and let $G_{1}$ and $G_{2}$ be the images of $G$ under the natural projections into $\operatorname{GL}\left(V_{1}\right)$ and $\mathrm{GL}\left(V_{2}\right)$. Suppose that $G_{1}$ has an abelian normal subgroup $A_{1}$ of index $N_{1}$ and that $G_{2}$ has an abelian normal subgroup $A_{2}$ of index $N_{2}$. Then $G$ has an abelian normal subgroup $A$ of index at most $N_{1} N_{2}$.

Proof. Viewing $\mathrm{GL}\left(V_{1}\right) \times \mathrm{GL}\left(V_{2}\right)$ as a subgroup of $\mathrm{GL}(V)$, we have a natural map from $G$ into $G_{1} / A_{1} \times G_{2} / A_{2}$ whose kernel $G \cap\left(A_{1} \times A_{2}\right)$ is abelian.

Theorem A follows immediately from this lemma. First, since the restriction of $G$ to $\operatorname{Rad}(\mathrm{B})$ is equivalent to a real orthogonal representation, this summand gives a factor $g(k)$. If B is skew symmetric, then the action of $G$ on $V_{0}$ embeds into $\operatorname{Sp}\left(V_{0}\right)$ and so contributes a factor $f(m)$ by Theorem B; if B is symmetric, then we further decompose $V_{0}$ as $V_{p} \oplus V_{q}$ by Theorem $\mathrm{C}$ and, by Lemma 7, obtain the contribution $g(p) \cdot g(q)$.

Acknowledgments. This paper combines pieces of work carried while the author was visiting the California Institute of Technology. He thanks the Institute for its support and Michael Aschbacher and David Wales for valuable conversations.

\section{Bibliography}

[1] A. Borel, Sous-groupes compacts maximaux des groups de Lie, Séminaire Bourbaki 1950/51, Exp. No. 33.

[2] M. J. Collins, On Jordan's theorem for complex linear groups, J. Group Theory 10 (2007), 411-423.

[3] M. J. Collins, Bounds for finite primitive complex linear groups, J. Algebra 319 (2008), 559-576.

[4] M. J. Collins, Induced representations and invariant forms, Israel J. Math. 197 (2013), 51-53.

[5] M. J. Collins, On finite subgroups of the classical groups, J. Group Theory 18 (2015), 511-534.

[6] C. W. Curtis and I. Reiner, Representation Theory of Finite Groups and Associative Algebras, Interscience, New York, 1962.

[7] S. Helgason, Differential Geometry, Lie Groups and Symmetric Spaces, Academic Press, New York, 1978.

[8] G. Hochschild, The Theory of Lie Groups, Holden-Day, San Francisco, 1965.

[9] J.-P. Serre, Linear Representations of Finite Groups (translated from the second French edition by Leonard L. Scott), Grad. Texts in Math. 42, Springer, New York, 1977. 
Received November 11, 2017; revised December 16, 2018.

\section{Author information}

Michael J. Collins, University College, Oxford OX1 4BH, United Kingdom.

E-mail: michael.collins@univ.ox.ac.uk 